Please do not remove this page

RMIT

UNIVERSITY

\title{
Kinetics of the lamellar gel-fluid transition in phosphatidylcholine membranes in the presence of sugars
}

Lenne, Thomas; Garvey, Chris; Koster, Karen; Bryant, Gary

https://researchrepository.rmit.edu.au/esploro/outputs/9921857814601341/filesAndLinks?institution=61RMIT_INST\&index=null

Lenne, T., Garvey, C., Koster, K., \& Bryant, G. (2010). Kinetics of the lamellar gel-fluid transition in phosphatidylcholine membranes in the presence of sugars. Chemistry And Physics Of Lipids, 163(2), 236-242. https://doi.org/10.1016/j.chemphyslip.2009.12.001

Document Version: Accepted Manuscript

Published Version: https://doi.org/10.1016/j.chemphyslip.2009.12.001

Repository homepage: https://researchrepository.rmit.edu.au

(C) 2010 Elsevier Ireland Ltd. All rights reserved

Downloaded On 2023/04/26 09:28:33 +1000 
Thank you for downloading this document from the RMIT Research Repository.

The RMIT Research Repository is an open access database showcasing the research outputs of RMIT University researchers.

RMIT Research Repository: http://researchbank.rmit.edu.au/

\section{Citation:}

Lenne, T, Garvey, C, Koster, K and Bryant, G 2010, 'Kinetics of the lamellar gel-fluid transition in phosphatidylcholine membranes in the presence of sugars', Chemistry And Physics Of Lipids, vol. 163, no. 2, pp. 236-242.

See this record in the RMIT Research Repository at:

http://researchbank.rmit.edu.au/view/rmit:5747

Version: Accepted Manuscript

Copyright Statement: (c) 2010 Elsevier Ireland Ltd. All rights reserved

Link to Published Version:

http://dx.doi.org/10.1016/j.chemphyslip.2009.12.001 


\title{
Kinetics of the lamellar gel-fluid transition in phosphatidylcholine membranes in the presence of sugars.
}

\author{
Thomas Lenné ${ }^{\mathrm{a} * \dagger}$, Christopher J. Garvey ${ }^{\mathrm{b}}$, Karen L. Koster ${ }^{\mathrm{c}}$ and Gary Bryant ${ }^{\mathrm{a}}$ \\ ${ }^{a}$ Applied Physics, RMIT University, Melbourne, Vic, 3001, Australia \\ ${ }^{\mathrm{b}}$ Australian Nuclear Science and Technology Organization, Menai, Australia \\ ${ }^{c}$ Department of Biology, The University of South Dakota, Vermillion, SD, USA
}

*Corresponding Author:

Tel: 61-2-6125 4061

Fax: 61-3-6125 5095

Email: Thomas.lenne@anu.edu.au

Keywords: phospholipids, lipid phase transitions, sugars, desiccation, freezing, small angle X-ray scattering.

\footnotetext{
${ }^{\dagger}$ Current address; Functional Ecology Group, Research School of Biological Sciences, The Australian National University, Canberra, ACT 0200, Australia.
} 


\begin{abstract}
Phase diagrams are presented for dipalmitoylphosphatidylcholine (DPPC) in the presence of sugars (sucrose) over a wide range of relative humidities $(\mathrm{RH})$. The phase information presented here, determined by small angle X-ray scattering (SAXS), is shown to be consistent with previous results achieved by Differential Scanning Calorimetry (DSC). Both techniques show a significant effect of sucrose concentration on the phase behaviour of this phospholipid bilayer. An experimental investigation into the effect of sugars on the kinetic behavior of the gel to fluid transition is also presented showing that increasing the sugar content appears to slightly increase the rate at which the transition occurs.
\end{abstract}




\section{Introduction}

On the subject of membrane damage due to freezing and desiccation a number of key points are generally considered to be resolved; 1 . The physical effects and consequent membrane damage that result from (slow) freezing can be very similar to those resulting from desiccation in air (reviewed in (Wolfe and Bryant, 1999)); 2. The plasma membrane is one of the primary sites of cellular damage due to both freezing and desiccation (reviewed in (Steponkus, 1984; Walters et al., 2001); 3. This damage can result from phase changes in the membrane bilayer (also reviewed in (Steponkus, 1984; Steponkus et al., 1995; Walters et al., 2001)); 4. Sugars can help stabilize membranes at low hydrations by lowering the temperature of these deleterious phase transitions (Crowe et al., 1984; Strauss et al., 1986; Koster et al., 2000; Koster et al., 2003; Bryant and Koster, 2004).

The mechanisms by which sugars affect phospholipid phase transition temperatures are becoming clearer, but there is still some confusion. This confusion lies partly in the fact that most studies of the effects of sugar have been carried out at very low or zero hydration (Crowe et al., 1984; Crowe et al., 1996; Cacela and Hincha, 2006). These studies have concluded that the effects of sugars on membrane phase transition temperatures are due, in part, to specific hydrogen bonding between lipid headgroups and sugars (Crowe et al., 1984; Crowe et al., 1996), with insertion of sugars between the headgroups a key aspect of some versions of this model (Crowe et al., 1996; Cacela and Hincha, 2006). However, studies of the effects of sugars at a range of hydrations suggest that the effects of sugars are primarily due to the osmotic and volumetric effects, and are non-specific (Koster et al., 2000). This explanation, which leads to a quantitative model that has been experimentally verified, is known as the hydration forces explanation (Bryant et al., 2001). In addition, to date, most studies have used high sugar concentrations (e.g., $>1: 1$ on a molar basis), which can lead to partial exclusion of the sugars 
and makes the quantitative interpretation of the data very difficult. See (Santivarangkna et al., 2008) for a recent review of some of this work.

In order to shed some light on this problem we have recently reported results using Small Angle Neutron Scattering (SANS) - contrast variation (Lenné et al., 2006), Small and Wide Angle X-ray Scattering SAXS/WAXS (Lenné et al., 2009) and Differential Scanning Calorimetry (DSC) (Lenné et al., 2007). This work has made significant progress in clarifying and quantifying the effects of sugars on the gel-fluid transition. In this paper we complete the current series of investigations by (i) presenting complete phase diagrams for one of the systems studied (sucrose-DPPC) and (ii) studying the effects of sucrose on the kinetics of the fluid-gel transition of a phosphatidylcholine, DPPC.

X-ray diffraction techniques, particularly SAXS, have been extensively used to determine the structure and phase of phospholipid bilayers in the presence of sugars (Caffrey et al., 1988; Tsvetkov et al., 1989; Nakagaki et al., 1992; Nagase et al., 1997, 1998; Demé et al., 2002). Many of these SAXS investigations have either been at a constant solute ratio or at constant hydration, and those solute ratios that have been used are often very high - typically 1 sugar per lipid or more. However it has recently been shown that only small concentrations (e.g. for a disaccharide, solute:lipid ratios $\leq 0.5: 1$ on a molar basis) are needed to have a significant effect on both transition temperatures (Lenné et al., 2007) and bilayer structure (Lenné et al., 2009), and the addition of extra solute has little further effect (Lenné et al., 2007). Moreover, excess sugar has been shown to be excluded from the interbilayer region (Demé et al., 2002; Lenné et al., 2006), consistent with recent simulations showing that sugars are excluded from the hydration layer near phospholipid headgroups (Westh, 2008).

One area that has not been fully investigated to date is whether sugars have any effect on the rate at which the fluid-gel transition occurs. Any effect on the rate of the transition may have additional implications regarding membrane integrity and cell survival, and may provide further 
evidence for the mechanism of solute protection. First, the presence of sugars will increase the viscosity, which might lead to a slight slowing of the transition. Second, and more importantly, if sugars insert between the lipid headgroups, as has been suggested in some models (Crowe et al., 1996; Patist and Zoerb, 2005), this should cause a significant slowing in the fluid-gel phase transition, as the area per headgroup needs to contract significantly during the transition. Although there have been a number of studies on the kinetics of the phase transitions of phospholipid bilayers (Caffrey and Bilderback, 1984; Caffrey, 1985; Tenchov et al., 1988), to our knowledge the only study of the effects of sugars on the kinetics of the fluid-gel transition is that of (Quinn et al., 1988). This study was limited to very low hydration (freeze dried) and two sugar concentrations. The results reported in the current paper are therefore the first study of the effects of varying the hydration and the sugar content on the kinetics of the fluid-gel transition.

Moreover, although a large amount of phase information for lipid bilayers in the presence of high sugar concentrations is available, mostly derived by DSC (Crowe et al., 1984; Nakagaki et al., 1992; Koster et al., 1994; Zhang and Steponkus, 1995; Zhang and Steponkus, 1996; Koster et al., 2000; Shalaev and Steponkus, 2001, 2001; Koster et al., 2003; Bryant and Koster, 2004), there has been a deficiency of data for low/intermediate sugar concentrations where it has been shown that the effects of sugars are significant (Lenné et al., 2007). Presented here are three phase diagrams derived by structural SAXS/WAXS measurements over a complete range of RH for three sucrose:lipid molar ratios, $0.1,0.2$ and $0.5: 1.0$.

\section{Experimental Procedures}

Sample preparation is described in (Lenné et al., 2009) and is briefly outlined here for completeness. Samples consisted of DPPC (1,2-dipalmitoylphosphatidylcholine) (Avanti Polar Lipids (Birmingham, AL, USA)) and sucrose (>99.5\% purity, Sigma Chemical Co. (St. Louis, 
MO, USA)). All were used without further purification. Dry DPPC was suspended in an appropriate amount of sugar solution to achieve the desired sugar:DPPC molar ratio. Further milli-Q water was added as necessary to ensure the samples were in excess water. Samples were mixed by repeated freeze-thawing, vortex mixing and centrifugation, then equilibrated at $23{ }^{\circ} \mathrm{C}$ over saturated salts that generate known Relative Humidities (RH) (Rockland, 1960; Young, 1967) for a period of 1-3 weeks. The RHs were monitored with a humidity data logger (Hastings, Port Macquarie, Australia) to ensure equilibrium had been reached. Once equilibrated, samples for X-ray analysis were transferred into $1.5 \mathrm{~mm}$ quartz X-ray capillaries (Wolfgang Muller Glas Technik, Berlin) and sealed using silicone (Pro Seal instant gasket, Racer Technology, USA). Sample homogeneity was confirmed by the existence of only a single set of SAXS scattering peaks and by the reproducibility of the SAXS results during repeated heating/cooling cycles.

Synchrotron Small and Wide angle X-ray scattering (SAXS and WAXS) experiments were carried out on the ChemMatCARS 15ID-D beamline at the Advanced Photon Source (APS), Argonne National Laboratory, Argonne, IL, U.S.A. X-rays scattering at an angle $2 \theta$ relative to the incoming beam correspond to a scattering vector $\mathrm{q}=\frac{4 \pi}{\lambda} \sin \theta$, where $\lambda$ is the wavelength. Regular structures in the sample of characteristic length d will lead to reflections given by the Bragg condition: $2 \mathrm{~d} \sin \theta=\mathrm{m} \lambda$, where $\mathrm{m}$ is the order of the reflection. Expressed in terms of $\mathrm{q}$, the repeat spacing is therefore given by: $d=\frac{2 \pi m}{q}$. For the experiments conducted here $\lambda=0.6199 \AA$ and the sample to detector distance was $555 \mathrm{~mm}$. Diffraction patterns were recorded on a Bruker $6000 \mathrm{CCD}$ detector over a range of scattering vectors, 0.046 to $1.7 \AA^{-1}$, covering the length scales of interest for the primary repeat distance and the wide angle reflection. For further details see (Cookson et al., 2006; Lenné, 2008).

A series of static measurements was made for each sample both below and above the gelto-fluid phase transition temperature at $10{ }^{\circ} \mathrm{C}$ increments between $20{ }^{\circ} \mathrm{C}$ (gel phase) and $70{ }^{\circ} \mathrm{C}$ 
(fluid phase). Following this a series of kinetic measurements was made by ramping the temperature rapidly through the phase transition whilst taking 1 second exposures every 9 seconds, resulting in an average of 20 measurements for each temperature scan. These kinetic runs were made between room temperature $\left(\sim 20^{\circ} \mathrm{C}\right)$ and $80{ }^{\circ} \mathrm{C}$ and with temperature both ascending and descending. The ramp rate of $\sim 15{ }^{\circ} \mathrm{C} / \mathrm{min}$ was calibrated using in situ thermocouples in a separate control experiment. This ramp rate is consistent with that used in the DSC experiments $\left(20{ }^{\circ} \mathrm{C} / \mathrm{min}\right)$ from which the DSC data in figure 3 are derived. Further details of these DSC experiments can be found in (Lenné et al., 2007; Lenné, 2008)

The data were radially averaged and processed by the IDL virtual machine software (ITT corp. Colorado, USA) using the SAXS15ID application written by David Cookson (ASRP/ChemMatCars). Data were exported into Excel (Microsoft Corp.) and then imported into either Origin (OriginLab Corporation, Northampton MA, USA.) for the static experiments, or Igor Pro software (WaveMetrics Inc. Oregon, USA) for the kinetic experiments, to obtain both the chain-chain and interlamellar repeat spacing peak positions. Experiment number was converted to temperature using thermocouple data stored in the log files then adjusted using the temperature calibration. Sigmoid fits were then applied to the data to quantify the effects of both humidity and sucrose:DPPC ratio on the characteristics of the transitions.

\section{Results}

Figure 1 shows two 2-dimentional scattering patterns that are typical of DPPC in the fluid (left) and gel (right) phases. The figure on the left is DPPC in the fluid phase at $70{ }^{\circ} \mathrm{C}$. The bright circular reflection nearest to the beam-stop (the dark spot in the centre) corresponds to the primary repeat spacing with two higher order reflections also present. Also visible at high $q$ is the peak due to the chain-chain spacing, which is quite diffuse. This is characteristic of lipids in the fluid phase, and is a result of the irregular, or fluid-like, 
arrangement of the acyl chains within the plane of the membrane. In the gel phase at $20{ }^{\circ} \mathrm{C}$ (figure on right) the structure of the gel phase is evident by both the sharpness of the chain-chain wide angle peak, and the increased number of higher order repeat reflections.

Figure 2 shows radial averages of the two dimensional scattering patterns from the two samples in figure 1. Here the distinction between the two phases is evident. First the chain-chain spacing peak is at a larger $q$ value and is much sharper in the gel phase (dashed line), relative to the diffuse peak of the fluid phase (solid line). The increased $q$ value corresponds to a decrease in the chain-chain spacing associated with the compression of the lipids in the gel phase. Second, the primary repeat spacing shifts to lower $q$ in the gel phase, corresponding to the increase in repeat spacing as the chains of the lipid are elongated. And third, there are more (and stronger) higher order reflections for the lipid in the gel phase relative to the fluid phase.

Determining the phase of the samples by their structural parameters at equilibrium provides complementary information to previous DSC measurements, which do not in themselves determine the phase of the sample, only the temperature of the transition between phases. By making X-ray measurements at a number of temperatures with samples prepared at varying $\mathrm{RH}$ and sugar content, the peak positions, in combination with a visual inspection of the shape of the chain-chain peaks, enable us to determine the phase of the samples. Figure 3 shows phase diagrams determined by SAXS/WAXS for DPPC with 3 sucrose:DPPC ratios; 0.1 (bottom), 0.2 (middle) and 0.5 (top). In each of these 3 graphs the transition temperatures determined by DSC are also shown in red (solid data markers). The (scanning) DSC data are in good agreement with the (equilibrium) SAXS data. Note that the DSC values and error bars represent the average of both heating and cooling scans, as described in (Lenné, 2008).

Figure 4 shows a typical example of a set of intensity versus scattering vector $(q)$ plots for a DPPC sample as temperature is scanned down from $70{ }^{\circ} \mathrm{C}$ to $20{ }^{\circ} \mathrm{C}$. The scans are displayed with $70{ }^{\circ} \mathrm{C}$ at the rear of the figure, down to $20{ }^{\circ} \mathrm{C}$ at the front. At either of the 
temperature extremes the plots are very similar to the equilibrium measurements presented in figure 2, and the same observation regarding the changes in the structure can be made. Now, however, in figure 4 the structural evolution of the sample between the two phases can also be observed. Again, the two areas of interest are the chain-chain reflection at approximately $q=1.4$ $-1.5 \AA^{-1}$, and the interlamellar repeat spacing reflection at approximately $q=0.1 \AA^{-1}$. The structural changes in the sample in each phase, and during the transition, are clearly observed.

To quantify these changes, the peak positions are determined and plots are made of the repeat and chain-chain peak positions as functions of temperature. Figure 5 shows an example of such a plot. The data are presented in this way to highlight the fact that the repeat spacing and the chain-chain peak show the same trends, and that the inflection points of both curves agree. In all data sets obtained, the chain-chain peak position data mirror the repeat spacing data, so the chain-chain data are not considered for further analysis.

There is a significant body of literature on the measurement of the kinetics of lipid phase transitions [eg (Caffrey and Cheng, 1995; Quinn, 1997; Cherezov et al., 2003) and references therein]. In most cases these papers applied temperature or pressure jumps to try to find the maximum transition speed, and used this to study the mechanisms of the transition. However, to our knowledge, there have been no attempts to fit the transition data - rather the transition is simply characterized by a transition duration [eg (Caffrey, 1989)]. Other workers have attempted to apply classical nucleation theory with some success [eg (Yang and Nagle, 1988; Kharakoz and Shlyapnikova, 2000)]. However, such analyses are model dependent, and too complex for the purposes of this study. Instead, we want to determine just two parameters from these data: the rate, $r$, of the transition between the two phases (expressed in units of $\AA / K$ or $\AA / s$ ); and the total duration of the transition, $\tau$. The rate $r$ can be estimated from the slope, but with so few points in the transition region, this is prone to error. The duration is difficult to measure unambiguously as 
one has to arbitrarily define a start and end point. In order to determine these parameters unambiguously, we fit the transition data using a generalized form of the sigmoid function:

$$
d(T)=d_{g}-\left(d_{g}-d_{f}\right)\left(1+\exp \left(\frac{T_{o}-T}{c}\right)\right)^{-1}
$$

where $d(T)$ is the repeat spacing as a function of temperature; $d_{g}$ is the $d$ spacing in the gel phase, $d_{f}$ is the $d$ spacing in the fluid phase, $T_{o}$ is the temperature at the inflection point, which occurs at $\mathrm{d}=\left(\mathrm{d}_{\mathrm{g}}+\mathrm{d}_{\mathrm{f}}\right) / 2$, and $\mathrm{c}$ is a fit parameter. The slope at the inflection point can be determined by differentiating with respect to $\mathrm{T}$, and setting $\mathrm{T}=\mathrm{T}_{\mathrm{o}}$, which yields the rate of transition $\mathrm{r}$ :

$$
r=\left|\frac{d_{g}-d_{f}}{4 c}\right|
$$

in units of $\AA / \mathrm{K}$. As the scanning rate is $15 \mathrm{~K} / \mathrm{min}=0.25 \mathrm{~K} / \mathrm{s}$, the transition rate can also be expressed as $r_{t}=0.25 * r$ in units of $\AA / s$. An unambiguous estimate of the duration $\tau$ of the transition can then be determined by the linear extrapolation from $d_{g}$ to $d_{f}$, which yields a duration of $\tau=\left(\mathrm{d}_{\mathrm{g}}-\mathrm{d}_{\mathrm{f}}\right) / \mathrm{r}_{\mathrm{t}}=16 \mathrm{c}$.

Figure 6 shows the results of cooling scans for samples equilibrated at $13 \% \mathrm{RH}$ for different sugar ratios. Each curve is fitted with the sigmoid function. Figure 7 shows the rate $r$ (left y-axis) and duration $\tau$ (right y-axis) as functions of sucrose:DPPC ratio for the data in figure 6. The duration shows no significant trend as a function of sugar ratio. However, the rate shows a significant trend, increasing from $\mathrm{r}=0.8 \AA / \mathrm{K}$ ( or $0.2 \AA / \mathrm{s}$ ) at low sugar content, up to $\mathrm{r}=2.0 \AA / \mathrm{K}$ (or $0.5 \AA / \mathrm{s}$ ) at a sucrose:lipid ratio of $1: 1$. In other words the presence of the sugar is increasing the rate at which the transition occurs. Of course there is an increase in the repeat spacing in the presence of sugar (Lenné et al., 2009). However, this result clearly shows that the presence of sugar is not slowing the transition from the fluid to the gel during cooling. Data for heating scans are very similar, and it is clear from figure 7 that there is no systematic difference in the speed of the transition (within the errors) for heating and cooling scans. This is in agreement with previous work (Quinn et al., 1988; Tenchov et al., 1988) which also showed no significant 
difference between the time spans of the heating and cooling transitions. This lends further support to the notion that the sugars are not inserted deep between the headgroups.

In the second set of experiments the effects of sample humidity on the kinetic characteristics of the gel:fluid transition were investigated using a set of constant sucrose:DPPC ratio (0.5) samples with varying humidity. Figure 8 shows the repeat spacing peak position during cooling for samples ranging in humidity from $0.1 \%$ to $91 \%$. In addition a sample was prepared in excess water and is labelled "XS".

The results for samples up to $57.5 \%$ humidity in figure 8 give good fits. In comparison with the data in figure 6 there is less variation in between the samples in both parameters (note the expanded scale). As these samples all have the same sucrose ratio, this is the expected result. However, the highest humidity samples show a completely different behaviour which was not observed in the static experiments. The repeat spacings for the three highest humidities all increase temporarily during cooling before decreasing again, finally ending at a slightly higher value in the gel phase than the fluid phase. Due to this behaviour, fits were not attempted for these three samples, which will be discussed later.

Figure 9 shows the rate $r$ (left y-axis) and duration $\tau$ (right y-axis) for the data in figure 8 . Within the scatter, the only trend that can be discerned is that the driest samples have the shortest duration, and therefore the fastest rate of transition. Again, if sugars were inserted between the headgroups, one would expect that as the membranes became drier the effect of the sugars would be to slow the transition, the opposite of what is observed.

\section{Discussion}

The phase diagrams determined by SAXS are in good quantitative agreement with the DSC results presented in (Lenné et al., 2007), so the discussion here will focus on the membrane structure and kinetics. 
Figure 6 shows that increasing the sugar:lipid ratio leads to an increase in the lamellar repeat spacing. This relationship implies, at least for the lower sugar:lipid ratios, that most of the sugar is incorporated into the aqueous region between the lamellae during dehydration, consistent with recent observations (Lenné et al., 2009). However, figure 6 also suggests that the increase in $\mathrm{d}$ spacing is not proportional to sugar concentration above 0.5 sucrose molecules per lipid. This is consistent with previous work which showed that some sugar is excluded into separate sugar:water microphases (Lenné et al., 2006). A consequence of this is that there is a limiting sugar:lipid ratio, beyond which the addition of further sugar has only a small effect on membrane transition temperatures (Lenné, 2008).

Figure 8 shows that at the lowest hydrations, the $d$ spacing is relatively unaffected by hydration, as expected since the bulk of the repeat spacing at low hydrations is due to the membrane thickness. The only unusual result observed is the behaviour of the high humidity samples during the transition, where the repeat spacings of all three samples consistently increased during the transition before dropping again. We have no definitive explanation for this effect at present, but pose the following two possible hypotheses: (i) in the coexistence region, it is well known that the lateral compressibility of membranes is greatly increased (i.e. their area modulus is greatly diminished), which can significantly increase the permeability. This may encourage mixing at these temperatures, redistributing water and/or sucrose between the intermembraneous and excluded microphases (Koster et al., 2003) during the transition; (ii) It is possible that there is a ripple phase in this temperature range. The ripple phase is a well known pre-transition phase observed in DPPC at high hydration (Wack and Webb, 1989). Although there was no evidence of the characteristic reflections of a ripple phase in our SAXS scans, it may be that the scan rate was too rapid for the transition to the ripple phase to occur fully, and the increase in $d$ spacing observed may be a consequence of this lack of equilibration. Further investigation of this effect will be the subject of future work. 
In the kinetic experiments the sample heating and cooling rates are relatively high consistent with ramp rates routinely used in a wide variety of experiments (e.g. DSC). The point of these experiments is not to reproduce rates of temperature change that occur in nature (which may be considerably slower), but rather to use this as a way of learning more about the mechanisms by which small sugars affect membrane phase transitions during dehydration.

Transitions from the fluid to gel phase occur as lipids are squeezed together by lateral compressive forces in the bilayer (Wolfe and Bryant, 1999). During this transition, the average surface area per lipid decreases on the order of 10-20 $\AA^{2}$ (Koster et al., 2000; Nagle and Tristram-Nagle, 2000; Lenné et al., 2009). Previous kinetic studies of the transition for pure DPPC indicate that this transition occurs within $2 \mathrm{~s}$ (Caffrey and Bilderback, 1984). One might expect that if sugar molecules were to intercalate between the adjacent lipid head groups, this would slow the transition, as the rearrangement of these larger molecules might be slower than that of water. In contrast, the data reported here show no systematic slowing of the transition rate in samples dried with increasing amounts of sucrose (Fig 7), and indeed the transition rate appears faster in the presence of sugars during both heating and cooling. This is in agreement with the kinetic studies of (Quinn et al., 1988), who showed for freeze-dried DPPC that the phase transition relaxation time was similar with or without trehalose, and suggested that the trehalose molecules form a "loosely packed 'monolayer' separating bilayers of phospholipid". This conclusion is also supported by (Lenné et al., 2009), who showed that there was no detectable spreading of the DPPC acyl chains when dried in the presence of sucrose.

Thus, these data support the view that sucrose molecules do not insert significantly between lipids in the membrane bilayer during the dehydration process. Interactions between sugars and lipids in dried systems are frequently modeled using some version of the Water Replacement Hypothesis (WRH), which posits direct hydrogen bonding between sugars and phospholipid as the necessary factor for membrane stabilization in the dry state (Crowe et al., 
1984; Clegg, 1986; Crowe et al., 1996; Crowe, 2007). In its simplest form, the WRH states that sugars form hydrogen bonds with membrane lipid headgroups and keep lipids laterally spaced in the fluid phase when water is removed. Some versions of the WRH suggest that the sugars intercalate between headgroups of adjacent lipids within the dried bilayer to keep the lipids spread apart in the fluid phase (e.g. (Crowe et al., 1996; Hoekstra et al., 2001; Crowe, 2007)), while other versions show the sugars bonding at the surface of the membrane (Sum et al., 2003). The data reported in the current work does not support the idea that there is deep insertion of the sugars into the membrane.

The hydration forces explanation (HFE) (Wolfe and Bryant, 1999; Koster et al., 2000; Bryant et al., 2001), in contrast, states that direct interactions between sugars and lipids are not necessary for bilayer stabilization, although they undoubtedly occur when most or all of the water has been removed. According to this explanation, the effects of sugars on membrane transition temperatures are attributed to the sugars' non-specific volumetric and osmotic effects. Moreover, the phase transition temperatures predicted by this model have been shown to be in quantitative agreement with experimental data (Lenné et al., 2009). The fact that sugars have virtually no effect on the bilayer structure (Sum et al., 2003; Lenné et al., 2009) or on the transition kinetics reported here, both lend further support to this explanation. These data are also consistent with the preferential exclusion of sugars from the hydration layers, as found in recent simulations (Westh, 2008).

Finally, we note that an additional facet of sugar-lipid interactions occurs if and when the sugar-rich solution vitrifies during dehydration. We previously demonstrated that sugar glasses can confer additional stability to dehydrated lipid bilayers (Koster et al., 1994; Koster et al., 2000; Bryant et al., 2001; Koster et al., 2003); however, the object of the current research was to avoid as much as possible the effects of glasses on the phase transition, and focus on the physical and volumetric effects of solutes. The choice of lipid (DPPC) and sucrose (rather than trehalose, 
which has a higher $\mathrm{T}_{\mathrm{g}}$ ), means that vitrification does not occur above the transition temperature of DPPC except (possibly) at the very lowest hydrations, and so vitrification should not play a role in the effects studied here. It is interesting, however, that the increased viscosity of the highly concentrated rubbery sugar solutions at the membrane surface did not systematically impact the rate of the lipid transition in these experiments.

\section{Conclusions}

The main conclusion from this investigation is that both dehydration and increasing sucrose content appear to increase (slightly) the rate of the transition between the fluid and gel phases of DPPC. The presence of sucrose does not slow the transition, but, if anything, speeds it up. These data lend support to the growing body of evidence that sugars do not penetrate deeply into the headgroup region at low (non-zero) hydrations. Further experiments, designed to unambiguously determine the location of the sugars, are currently underway.

These data do not support the existence of deep penetration of the sugar into the headgroup region of the lipids during dehydration.

\section{Acknowledgements}

Lenné and Bryant would like to thank Bob Shanks for the use of the calorimeter, David Cookson for expert help with the synchrotron experiments and Vincent Martinez for help with the Igor Pro macros. This work, including use of the ChemMatCARS sector, was supported by the Australian Synchrotron Research Program, which is funded by the Commonwealth of Australia under the Major National Research Facilities Program. Use of the Advanced Photon Source was supported by the U. S. Department of Energy, Office of Science, Office of Basic Energy Sciences, under Contract No. DE-AC02-06CH11357. 
Bryant G, Koster KL (2004) Dehydration of solute - lipid systems: hydration forces analysis. Colloids and Surfaces B: Biointerfaces 35: 73 - 79

Bryant G, Koster KL, Wolfe J (2001) Membrane behaviour in seeds and other systems at low water content: the various effects of solutes. Seed Science Research 11: 17-25

Cacela C, Hincha DK (2006) Low Amounts of Sucrose Are Sufficient to Depress the Phase Transition Temperature of Dry Phosphatidylcholine, but Not for Lyoprotection of Liposomes. 90: 2831-2842

Caffrey M (1985) Kinetics and mechanisms of the lamellar gel/lamellar liquid-crystal and lamellar/inverted hexagonal phase transition in phosphatidylethanolamine: a real-time xray diffraction study using synchrotron radiation. Biochemistry 24: 4826-4844

Caffrey M (1989) The Study of Lipid Phase-Transition Kinetics by Time-Resolved X-RayDiffraction. Annual Review of Biophysics and Biophysical Chemistry 18: 159-186

Caffrey M, Bilderback DH (1984) Kinetics of the main phase transition of hydrated lecithin monitored by real-time X-ray diffraction. Biophys. J. 45: 627-631

Caffrey M, Cheng A (1995) Kinetics of lipid phase changes. Current Opinion in Structural Biology 5: 548-555

Caffrey M, Fonseca V, Leopold AC (1988) Lipid-Sugar Interactions: Relevance to Anhydrous Biology. Plant Physiology 86: 754-758

Cherezov V, Siegel DP, Shaw W, Burgess SW, Caffrey M (2003) The kinetics of non-lamellar phase formation in DOPE-Me: Relevance to biomembrane fusion. Journal of Membrane Biology 195: 165-182

Clegg JS (1986) The physical properties and metabolic status of Artemia cysts at low water contents: the Water Replacement Hypothesis. In AC Leopold, ed, Membranes, Metabolism, and Dry Organisms. Cornell University Press, Ithaca, NY., pp 169-187

Cookson D, Kirby N, Knott R, Lee M, Schultz D (2006) Strategies for data collection and calibration on the small angle x-ray scattering camera at ChemMatCars, Advanced Photon Source. Journal of Synchrotron Radiation 13: 440 - 444

Crowe JH (2007) Trehalose as a "chemical chaperone": fact and fantasy. . In P Csermely, L Vigh, eds, Molecular aspects of the stress response: chaperones, membranes and networks. . Springer, New York, pp 143-158

Crowe JH, Crowe LM, Chapman D (1984) Preservation of Membranes in Anhydrobiotic Organisms: The Role of Trehalose. Science (Washington, D. C., 1883-) 223: 701-703

Crowe JH, Hoekstra FA, Nguyen KHN, Crowe LM (1996) Is vitrification involved in depression of the phase transition temperature in dry phospholipids? Biochimica et Biophysica Acta 1280: 187-196

Demé B, Dubois M, Zemb T (2002) Swelling of a lecithin lamellar phase induced by small carbohydrate solutes. Biophysical Journal 82: 215-225

Hoekstra FA, Golovina EA, Buitink J (2001) Mechanisms of plant desiccation tolerance. Trends in Plant Science 6: 431-438

Kharakoz DP, Shlyapnikova EA (2000) Thermodynamics and kinetics of the early steps of solid-state nucleation in the fluid lipid bilayer. Journal of Physical Chemistry B 104: 10368-10378

Koster KL, Lei YP, Anderson M, Martin S, Bryant G (2000) The effect of Vitrified and Nonvitrified Sugars on Phosphatidylcholine Fluid-to-Gel Phase Transitions. Biophysical Journal 78: 1932-1946

Koster KL, Maddocks KJ, Bryant G (2003) Exclusion of maltodextrins from phosphatidylcholine multilayers during dehydration: effects on membrane phase behaviour. European Biophysics Journal 32: 96-105 
Koster KL, Webb MS, Bryant G, Lynch DV (1994) Interactions between soluble sugars and POPC (1-palmitoyl-2-oleoyl-phosphatidylcholine) during dehydration: vitrification of sugars alters the phase behaviour of the phospholipid. Biochimica et Biophysica Acta 1193: $143-150$

Lenné T (2008) The effects of solutes on the phase behaviour of phospholipid membranes. . PhD. RMIT University, Melbourne

Lenné T, Bryant G, Garvey CJ, Keiderling U, Koster KL (2006) Location of sugars in multilamellar membranes at low hydration. Physica B: Condensed Matter

Proceedings of the Eighth International Conference on Neutron Scattering 385-386: 862-864

Lenné T, Bryant G, Holcomb R, Koster KL (2007) How much solute is needed to inhibit the fluid to gel membrane phase transition at low hydration? Biochimica et Biophysica Acta 1768: 1019 - 1022

Lenné T, Garvey CJ, Koster KL, Bryany G (2009) The Effects of Sugars on Lipid Bilayers during Dehydration - SAXS/WAXS Measurements and Quantitative Model. The Journal of Physical Chemistry 113: 2486-2491.

Nagase H, Ueda H, Nakagaki M (1997) Effect of water on lamellar structure of DPPC/sugar systems. Biochimica et Biophysica Acta (BBA) - Biomembranes 1328: 197-206

Nagase H, Ueda H, Nakagaki M (1998) Temperature change of the lamellar structure of DPPC/disaccharide/water systems with low water content. Biochimica et Biophysica Acta (BBA) - Biomembranes 1371: 223-231

Nagle JF, Tristram-Nagle S (2000) Structure of Lipid Bilayers. Biochimica et Biophysica Acta 1469: $159-195$

Nakagaki M, Nagase H, Ueda H (1992) Stabilization of the lamellar structure of phosphatidylcholine by complex formation with trehalose. Journal of Membrane Science 73: $173-180$

Patist A, Zoerb H (2005) Preservation mechanisms of trehalose in food and biosystems. Colloids and Surfaces B: Biointerfaces 40: 107-113

Quinn PJ (1997) Measurement of kinetics and mechanisms of phase transitions in lipid-water systems. Journal of Applied Crystallography 30: 733-738

Quinn PJ, Koynova RD, Lis LJ, Tenchov BG (1988) Lamellar gel-lamellar liquid crystal phase transition of dipalmitoylphosphatidylcholine multilayers freeze-dried from aqueous trehalose solutions. A real-time X-ray diffraction study. Biochimica et Biophysica Acta (BBA) - Biomembranes 942: 315-323

Rockland LB (1960) Saturated Salt Solutions for Static Control of Relative Humidity between $5^{\circ}$ and $40^{\circ} \mathrm{C}$. Analytical Chemistry 32: $1375-1376$

Santivarangkna C, Higl B, Foerst P (2008) Protection mechanisms of sugars during different stages of preparation process of dried lactic acid starter cultures. Food Microbiology 25: 429-441

Shalaev EY, Steponkus PL (2001) Depression of the Glass Transition Temperature of Sucrose Confined in a Phospholipid Mesophase. Langmuir 17: 5137-5140

Shalaev EY, Steponkus PL (2001) Phase behaviour and glass transition of 1,2dioleoylphosphatidylethanolamine (DOPE) dehydrated in the presence of sucrose. Biochimica et Biophysica Acta 1514: 100-116

Steponkus PL (1984) Role of the Plasma Membrane in Freezing Injury and Cold Acclimation. Annual Review of Plant Physiology 35: 543-584

Steponkus PL, Uemura M, Webb MS (1995) Freeze-induced destabilization of cellular membranes and lipid bilayers. In EA Disalvo, SA Simon, eds, Permeability and Stability of Lipid Bilayers. CRC Press, Boca Raton, pp 77-104 
Strauss G, Schurtenberger P, Hauser H (1986) The interaction of saccharides with bilayer vesicles: stabilization during freeze-thawing and freeze-drying. Biochimica et Biophysica Acta 858: 169-180

Sum AK, Faller R, Pablo JJd (2003) Molecular Simulation Study of Phospholipid Bilayers and Insights of the Interactions with Disaccharides. Biophysical Journal 85: 2830-2844

Tenchov BG, Lis LJ, Quinn PJ (1988) Structural rearrangements during crystal-liquid-crystal and gel-liquid-crystal phase transitions in aqueous dispersions of dipalmitoylphosphatidylethanolamine. A time-resolved X-ray diffraction study. Biochimica et Biophysica Acta (BBA) - Biomembranes 942: 305-314

Tsvetkov TD, Tsonev LI, Tsvetkova NM, Koynova RD, Tenchov BG (1989) Effect of Trehelose on the Phase Properties of Hydrated and Lyophylised Dipalmitoylphosphatidylcholine Multilayers. Cryobiology 26: 162-169

Wack DC, Webb WW (1989) Synchrotron X-ray study of the modulated lamellar phase P beta ' in the lecithin-water system. Physical Review A 40: 2712

Walters C, Farrant JM, Pammenter NW, Berjak P (2001) Desiccation stress and damage. In M Black, HW Pritchard, eds, Desiccation and Plant Survival. CABI Publishing, Oxford, pp 263-291

Westh P (2008) Glucose, sucrose and trehalose are partially excluded from the interface of hydrated DMPC bilayers. Physical Chemistry Chemical Physics 10: 4110-4112

Wolfe J, Bryant G (1999) Freezing, drying and/or vitrification of membrane-solute-water systems. Cryobiology 39: 103-129

Yang CP, Nagle JF (1988) Phase-Transformations in Lipids Follow Classical Kinetics with Small Fractional Dimensionalities. Physical Review A 37: 3993-4000

Young JF (1967) Humidity control in the laboratory using salt solutions-a review. Journal of Applied Chemistry 17: 241-245

Zhang J, Steponkus PL (1995) Effects of sugars on the dehydration-induced increase in the Tm of DPPC dehydrated over a continuum of osmotic pressures. Cryobiology 32: 60A

Zhang J, Steponkus PL (1996) Proposed mechanism for depression of the liquid-crystalline-togel phase transition temperature of phospholipids in dehydrated sugar-phospholipid mixtures. Cryobiology 33: 21A 


\section{Figure 1}

2-dimensional x-ray diffraction patterns from DPPC in the fluid phase at $70{ }^{\circ} \mathrm{C}$ (left), and in the gel phase at $20^{\circ} \mathrm{C}$ (right).

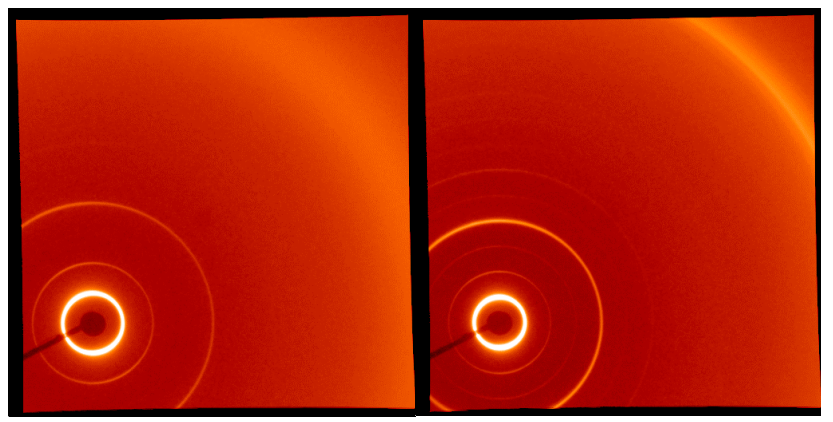

\section{Figure 2}

Intensity versus scattering vector plots for the 2-D diffraction patterns shown in figure 1.

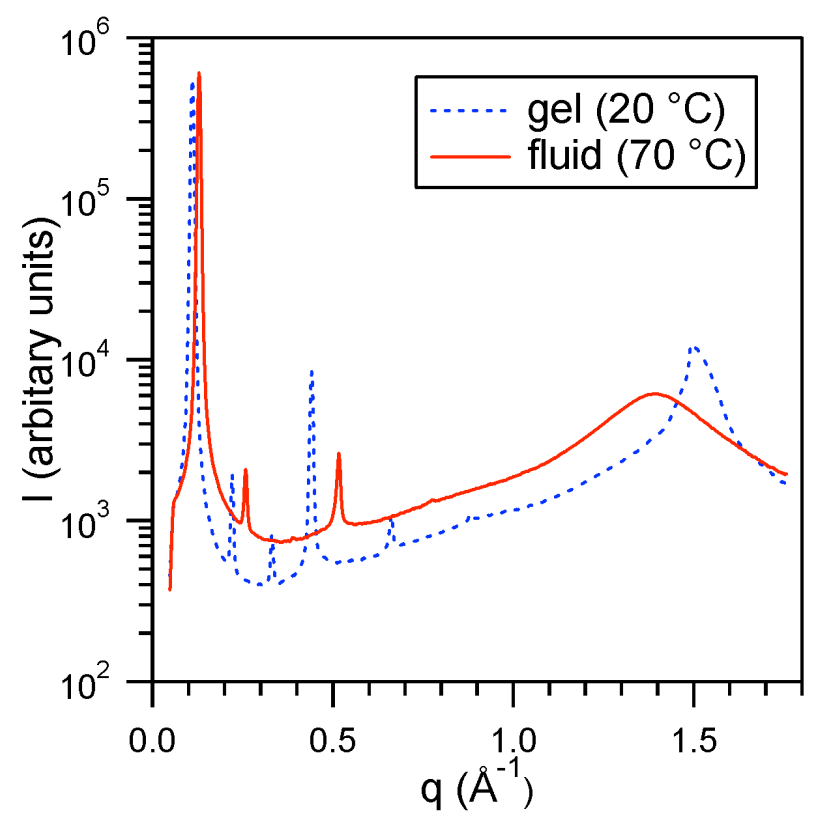




\section{Figure 3}

Phase diagrams determined by SAXS/WAXS for DPPC with 0.1 (bottom), 0.2 (middle) and 0.5 (top) sucrose:DPPC ratios in comparison with the transition temperatures determined by DSC (Lenné et al., 2007). The phases are gel (open circles), fluid (crosses) and coexistence (triangles). The solid circles correspond to the phase transition temperatures determined by DSC.

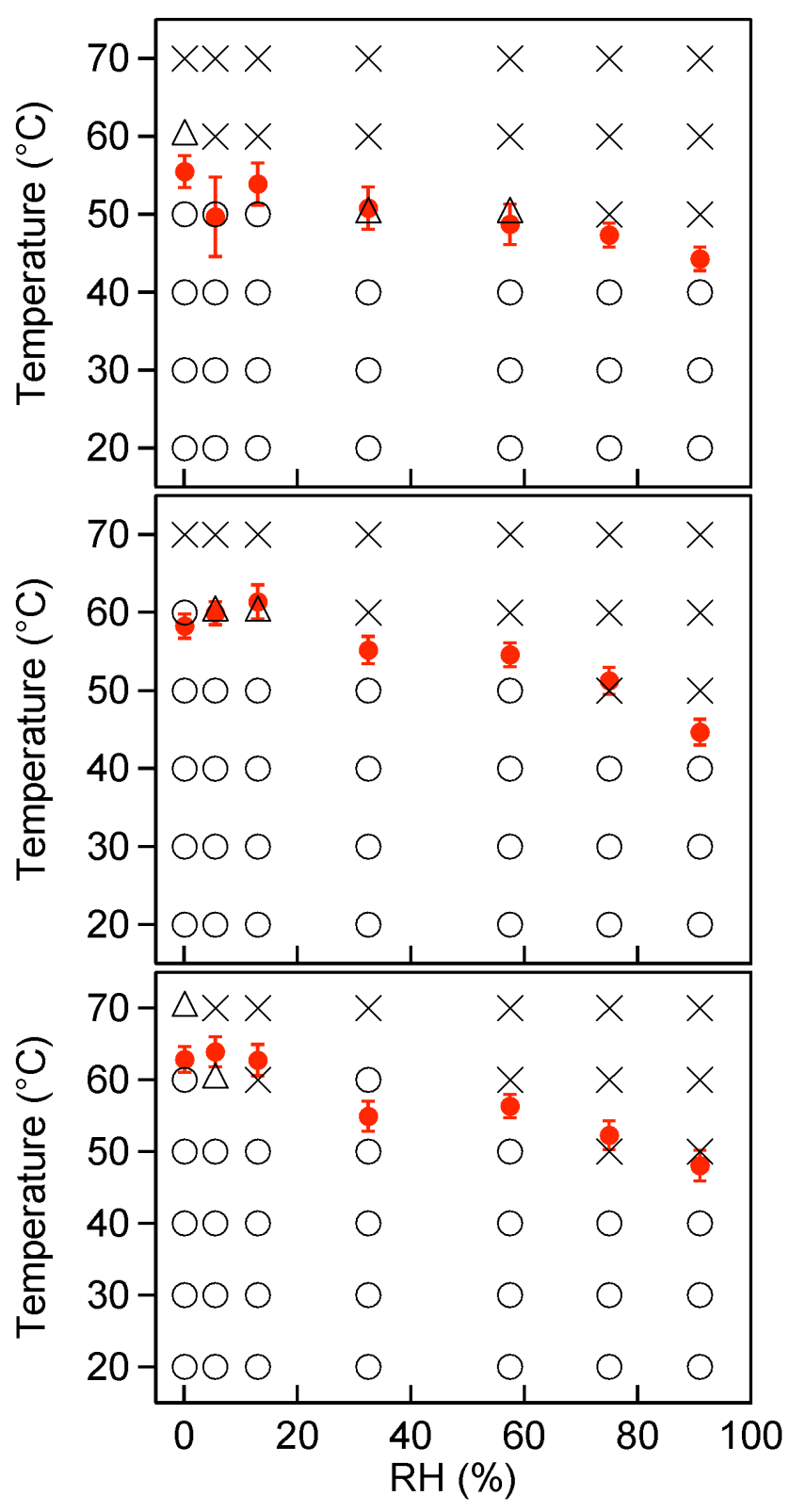




\section{Figure 4}

An example of a typical series of intensity versus scattering vector plots as temperature is scanned down from $70{ }^{\circ} \mathrm{C}$ (rear of figure) to $20{ }^{\circ} \mathrm{C}$ (foreground). (DPPC at $13 \% \mathrm{RH}$ with 0.1 sucrose:lipid ratio). Note that the traces are arbitrarily offset on the $\mathrm{y}$-axis and that every second plot has been removed in order better visualize the changes in the traces between the two phases.

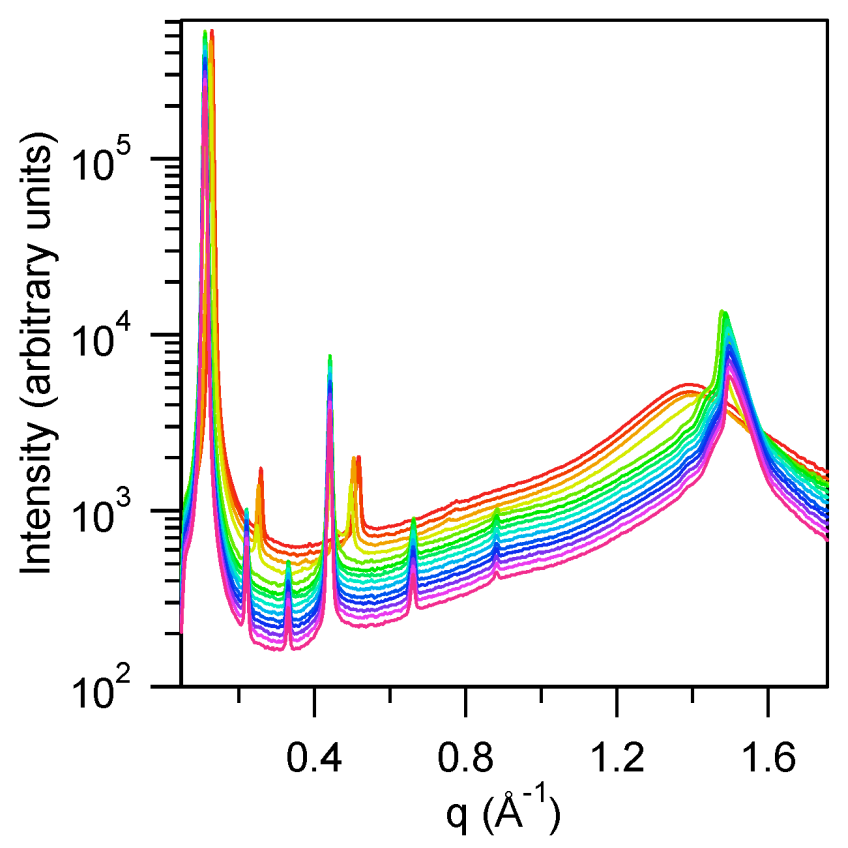

\section{Figure 5}

An example of interlamellar repeat and chain-chain spacings during a cooling scan at $\sim 15{ }^{\circ} \mathrm{C} / \mathrm{min}$. The interlamellar repeat spacings are shown by the solid circles (left $\mathrm{y}$ - axis), while the open circles show the chain-chain spacings (right $\mathrm{y}-$ axis). (DPPC at 13\% RH with 0.1 sucrose:lipid ratio).

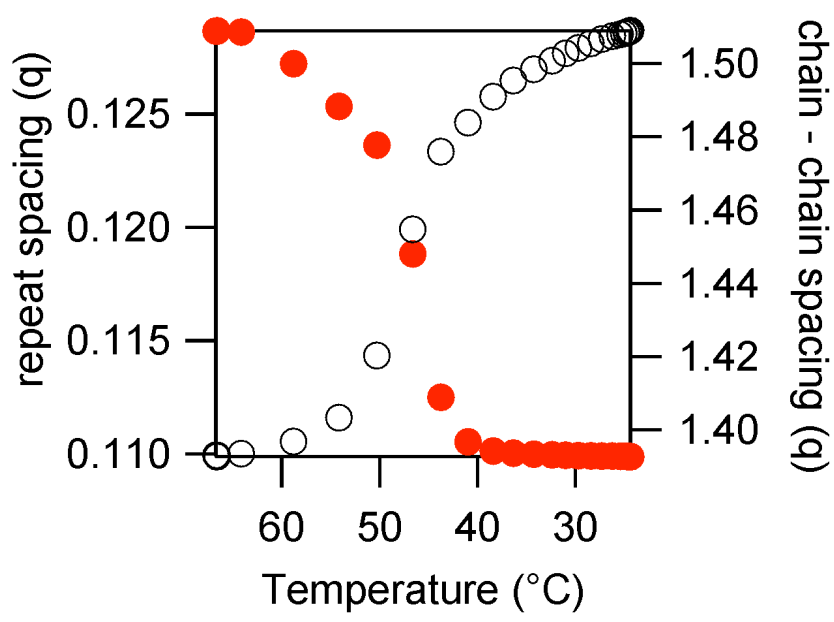




\section{Figure 6}

Interlamellar repeat spacings for DPPC at constant humidity $(13 \%)$ while scanning from $70{ }^{\circ} \mathrm{C}$ to 20 ${ }^{\circ} \mathrm{C}$ at $\sim 15{ }^{\circ} \mathrm{C} / \mathrm{min}$. Sugar:lipid ratios shown in the legend, and the lines are sigmoidal fits. Note that the experiment ran from right to left in this figure.

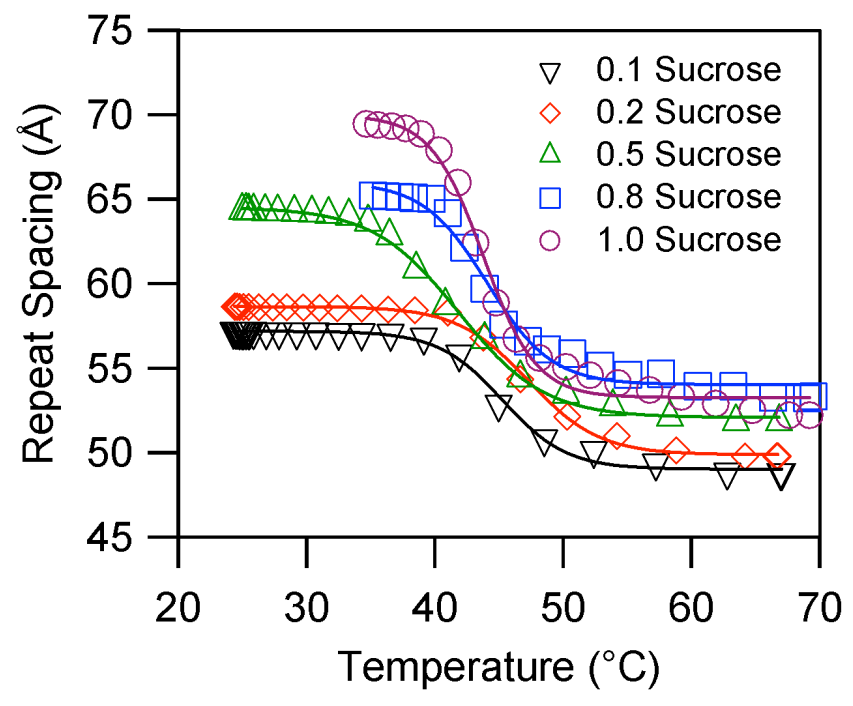

\section{Figure 7}

The parameters $m$ and $r$ from the sigmoid fits in figure 6 (DPPC at constant humidity, $13 \%$ ). The direction of the temperature scan is given by the legend. Errors in the fitting of the sigmoid functions are smaller than the size of the data markers.

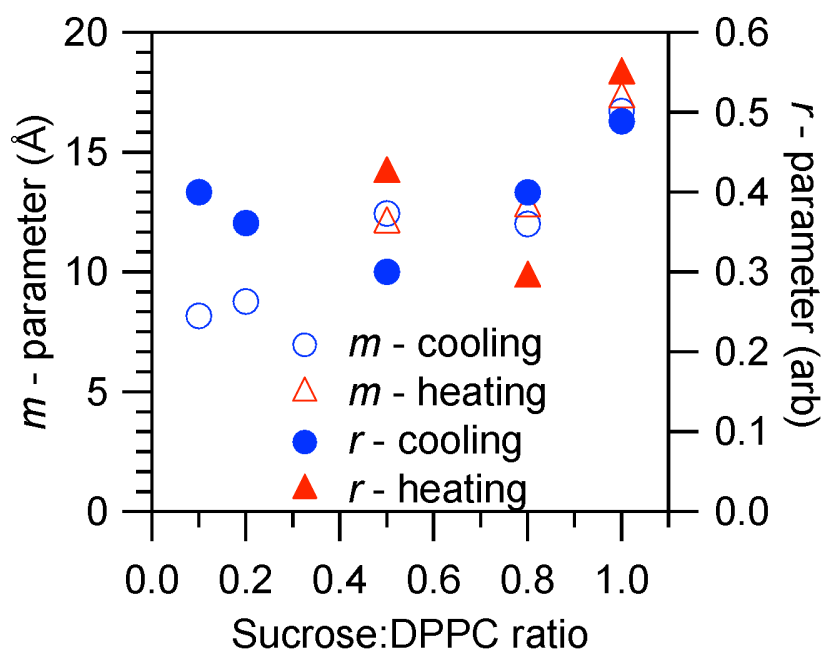




\section{Figure 8}

Repeat spacing for DPPC at constant sucrose:DPPC ratio $(0.5)$ while cooling from $70{ }^{\circ} \mathrm{C}$ to $20{ }^{\circ} \mathrm{C}$ through the fluid-to-gel transition at $\sim 15{ }^{\circ} \mathrm{C} / \mathrm{min}$. The repeat spacings are given by the data markers and the sigmoid fits with solid lines. The RH (\%) of the samples is given by the legend. Note that the experiment ran from right to left in this figure. "XS" refers to a sample prepared in excess water.

\section{Figure 9}

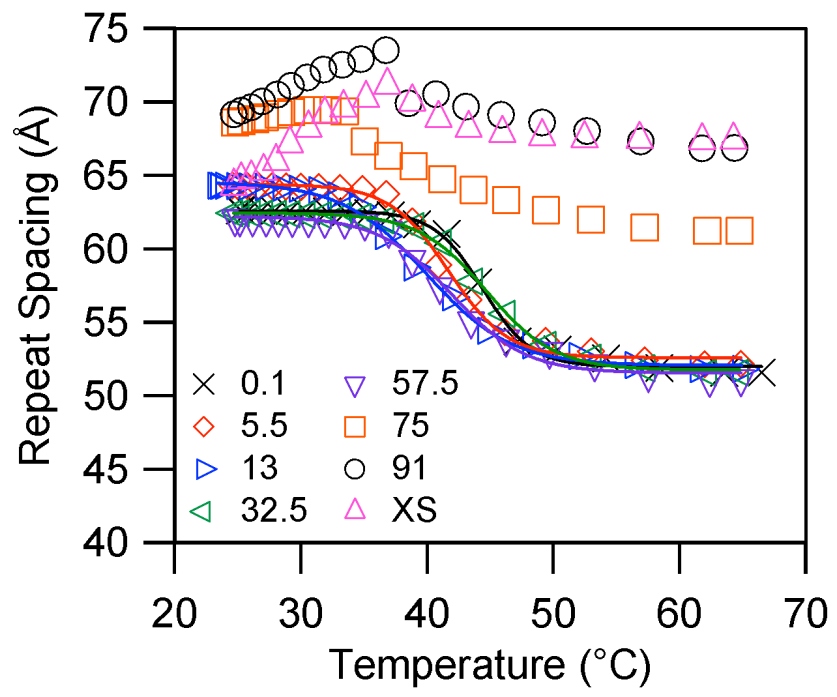

The parameters $m$ and $r$ from the sigmoid fits in figure 8 (DPPC at constant sucrose:lipid ratio, 0.5). The direction of the temperature scan is given by the legend. Errors in the fitting of the sigmoid functions are smaller than the size of the data markers. Note that the $m$ - cooling $32.5 \% \mathrm{RH}$ data marker is obscured by the $r$ - cooling $32.5 \% \mathrm{RH}$ data marker.

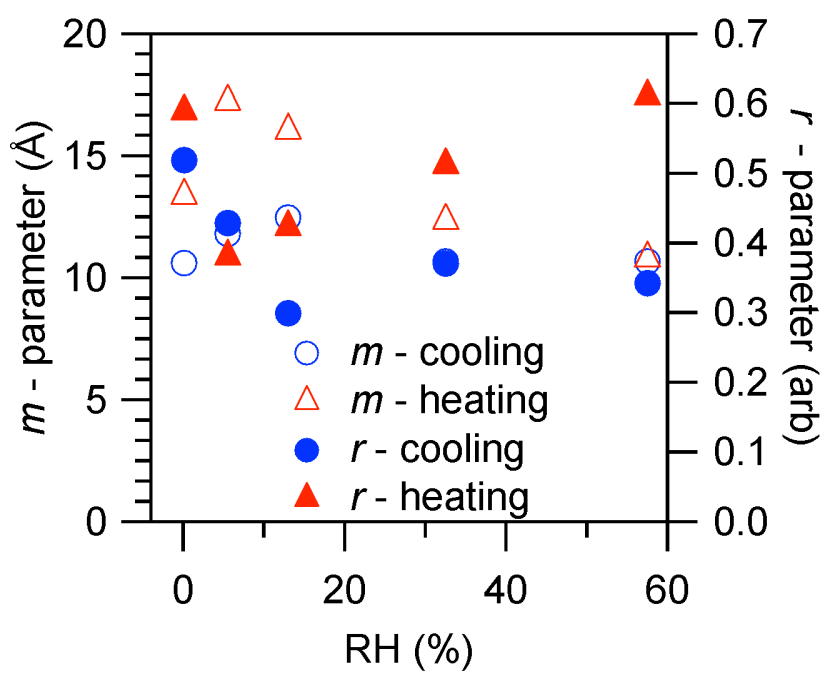

